\title{
Alcohol-Related Social Gatherings with Coworkers: Intentions to Behave and Intentions to Not Behave
}

\author{
Hee Sun Park' and Dong Wook Lee ${ }^{2}$ \\ ${ }^{1}$ Michigan State University, East Lansing, Michigan, USA \\ 2 Jeju National University, Jeju, Korea
}

\begin{abstract}
Two studies are reported using the theory of planned behaviour (TPB) to examine Koreans' intentions and self-reported behaviour of joining and not joining alcohol-related social gatherings with work colleagues after work hours. Study 1 showed that group-related attitudes and perceived behavioural control of the TPB components regarding joining were significantly associated with intentions to join. Study 2 examined TPB components regarding not joining and intentions to not join. Study 2 found that although none of the TPB components was directly related to intentions, subjective norms from work colleagues moderated the effect of group-related attitudes on intentions to not join. The TPB components and intentions were not significantly related to self-reported behaviour of joining. The findings indicate that the relationships between the TPB components and intentions can be different for engaging and not engaging in a behaviour.
\end{abstract}

Keywords: attitudes, subjective norms, perceived behavioural control, intentions to behave, intentions to not behave, South Korea

Various reasons likely exist for individual participation or nonparticipation in social gatherings after work hours, especially when the social gatherings often involve a moderate to heavy consumption of alcohol. Obtaining information useful for work and talking openly with coworkers can be reasons for participating in these gatherings. Wanting to go home on time or not wanting to get drunk can be reasons for not participating in these gatherings. Normative pressure from coworkers also can be a reason for either participation or nonparticipation.

The current study has two main purposes. First, with a sample of Korean working adults, this study tests the utility of the theory of planned behaviour (TPB) for predicting two related behaviours (here, joining versus not joining alcohol-related social gatherings). Second, considering that the attitudinal component of TPB is behavioural-outcome-based, the current study asks which types of attitudes are important for intentions to join or to not join alcoholrelated social gatherings.

\section{The Theory of Planned Behaviour}

The theory of planned behaviour (TPB) delineates attitudes toward behaviours, subjective norms, and perceived behavioural control that contribute to intentions and behaviours (Ajzen, 1988). TPB assumes that people examine the likelihood of outcomes that can result from the behaviour in question and evaluate importance of those outcomes. The behavioural outcomes and the evaluation of the outcomes are multiplied together to form attitudes toward behaviours. TPB postulates that people hold normative beliefs about what their important others (i.e., reference groups) think about performing behaviours and different degrees of motivation to comply with what their important others prefer. Normative beliefs and motivation to comply are multiplied with each other to form subjective norms. The third component, perceived behavioural control, refers to the extent to which individuals feel that they can engage in the behaviour. In other words, perceived behavioural control indicates individual efficacy levels in performing the behaviour. 
Meta-analyses findings have been generally supportive of TPB (e.g., Albarracín, Johnson, Fishbein, \& Muellerleile, 2001; Armitage \& Conner, 2001; Godin \& Kok, 1996). The theory has been useful for understanding the intentions and behaviours of people from nonwestern cultures (e.g., Chau \& Hu, 2001; Fekadu \& Kraft, 2002; Hu \& Lanese, 1998; Yun \& Park, 2010). TPB has been applied to alcohol-related intentions and behaviours (Hagger, Anderson, Kyriakaki, \& Darkings, 2007; Murgraff, McDermott, \& Walsh, 2001), although attitudes, compared to subjective norms, were more important for amount of self-reported drinking (Murgraff et al., 2001), for intention to seek help for alcohol abuse (Codd \& Cohen, 2003) and for engaging in heavy drinking (Norman, Bennett, \& Lewis, 1998).

As an extension of Ajzen and Fishbein's (1980) theory of reasoned action (TRA), TPB included perceived behavioural control as an additional predictor of intentions and behaviours. While TRA is limited to understanding and predicting volitional behaviours (Ajzen \& Fishbein, 1980), the perceived behavioural control component of TPB allows researchers to examine the extent to which individuals' internal and external control factors - such as capability, skills, resources, opportunities and barriers - are related to behavioural intentions and behaviours (Ajzen, 1988). For a behaviour of joining (or not joining) alcoholrelated social gatherings, individuals may differ in their perceived control levels in dealing with their work colleagues' invitation to gatherings. Thus, TPB, rather than TRA, can be more useful for the current study that examines Korean working adults' participation in alcohol-related social gatherings.

\section{TPB for Engaging Versus Not Engaging in a Behaviour}

When applying a theory to understand and predict an intention and a behaviour, it may be useful to examine two aspects of a behaviour: engaging in the behaviour versus not engaging in the behaviour. Sheppard, Hartwick and Warshaw's (1988) meta-analysis results showed that the relationship between intentions and behaviours was actually stronger (i.e., an average correlation of .77) for behaviours involving a choice among alternatives than for behaviours involving no choice (i.e., an average correlation of .47) and that attitudinal and normative components were well predictive of both intentions involving a choice among alternatives (i.e., an average correlation of .69) and intentions involving no choice (i.e., an average correlation of .64). Although engaging in the behaviour and not engaging in the behaviour can represent a type of alternative behaviours involving a choice, it is still unclear whether behavioural outcomes of one behaviour (i.e., engaging) would still be relevant to the other behaviour (i.e., not engaging). When the attitudinal component of TPB is found to be significantly related to intentions of, for example, exer- cising or consuming an excessive amount of alcohol, such findings are only about engaging in such behaviours, while providing little information about not engaging in such behaviours. For example, the outcome of having fun can be likely (i.e., the likelihood of the outcome) and important (i.e., the evaluation of the outcome) when drinking alcohol, but when people intend to not drink alcohol, it is questionable whether they form such intentions because they consider the outcome of having fun as unlikely and unimportant or because they consider the outcome of not having fun likely and important. It is necessary to empirically examine whether the presence or absence of certain behavioural outcomes is responsible for both engaging and not engaging in a behaviour.

\section{Alcohol-Related Social Gatherings in South Korea}

The prevalence of alcohol-related problems in South Korea necessitates examination of factors affecting participation and nonparticipation in alcohol-related social gatherings. According to a 2001 report produced by the Ministry of Health and Welfare (2003) in South Korea, $30.6 \%$ of adults (63.4\% of drinkers) belong to the category of drinkers who regularly consume amounts of alcohol considered harmful to health, and $48.9 \%$ of male drinkers belong to the category of high-risk drinkers. According to a 2002 statistical report by the Korea National Statistical Office (2003), liver-related diseases are the fourth most frequent death cause for Korean men in general and the second ranked cause of death among Korean men aged 40 to 49 . Because a significant portion of liver-related diseases are induced by alcohol consumption (Corrao, Bagnardi, Zambon, \& Arico, 1999; National Institute on Alcohol Abuse and Alcoholism, 1998), it can be inferred that alcohol poses a serious risk to working adults in South Korea.

One of the reasons for alcohol-related problems is that Korean working adults' work lives often extend to after-hours social gatherings with work colleagues. Alcohol-related social gathering with coworkers is often called 회식 (pronounced as 'hweasik'). Korean workers participate in social gatherings with their work colleagues on a regular basis (Lee, Park, Lee, Kim, \& Kim, 2007). Treating alcohol as a social lubricant, many Koreans hold the beliefs that alcohol helps people to get to know each other better and that an inability to drink can hurt an individual's social and work lives (Choi, Kim, \& Kim, 2001).The importance of maintaining amicable social and work relationships with coworkers and immediate supervisors is obvious, especially in Korean society, where hierarchy and custom are all-important (Cho \& Yoon, 2002). Direct and indirect social pressure is one of strong reasons for drinking among Korean college students (Sung \& Kim, 2009). Reasons for not being able to refuse requests to drink alcohol at 회식 include avoiding being a killjoy and supervisors being 
the requesters (Lee, 2009). People in higher status (e.g., supervisors, older person) may implicitly or explicitly pressure those in lower status to join social gatherings and drink alcohol. Koreans are more likely to drink with friends, colleagues and business associates rather than alone and drinking is an important way of developing social relationships (Kim, 1986).

Given that alcohol-related social gatherings are a part of social and organisational life, examination of the reasons for joining or not joining alcohol-related social gatherings can show that joining social gatherings with work colleagues is not just about consuming alcohol together, but more about social and organisational influences on individuals' intentions and behaviours. Korean cultural characteristics can be reflected in the TPB components regarding intentions to join and intentions to not join social gatherings. For example, a subjective norm may show social pressure that subordinates may perceive when supervisors suggest participation in social gatherings. Attitudes toward behaviour may include evaluations of the outcomes relevant to social and work relationships that can result from joining and not joining social gatherings. Perceived behavioural control may indicate the extent to which workplace climate affect Korean workers' perceptions about their own ability and skill in joining and not joining.

\section{Research Questions for Studies 1 and 2}

The current study is exploratory, posing three research questions regarding types of attitudes, intentions to join and to not join, and the relationship between intentions and self-reported behaviours. Certain types of attitudes may become more important for intentions to join or to not join alcohol-related social gatherings, as individuals may evaluate certain outcomes more favourably and consider them to be more likely to occur. Additionally, as the attitudinal, normative and perceived control components of TPB encompass different reasons for joining or not joining, these different components may be weighted differently in their relationships to intentions to join or to not join alcohol-related social gatherings. Furthermore, it is informative to examine whether intention to join and intention to not join are similarly or differently predictive of the behaviour of joining alcohol-related social gatherings.

A meta-analysis of ten meta-analyses with correlational data (Sheeran, 2002) and a meta-analysis of experimental evidence (Webb \& Sheeran, 2006) showed strong evidence that intentions are predictive of behaviours. This substantial body of empirical evidence and the TPB's proposition on the relationship between intentions and behaviours (Ajzen, 1988) may lead to an expectation that intentions to join and to not join would predict the self-reported behaviour of joining alcoholrelated social gatherings. On the other hand, a variety of factors (e.g., behaviour types, measurement artefacts, etc.) could influence the strength of the relationship between intentions and behaviours (Sheeran, 2002; Webb \& Sheeran, 2006). Collectivistic people may consider situational, external or relational factors as more important than their own attitudes or intentions when engaging in a behaviour (Kim, 2002). The current study used Korean working adults as the sample and examined the behaviour that involved gatherings with work colleagues. In sum, both studies 1 and 2 investigated the three research questions below:

- RQ1: Which types of attitudes toward behaviour are significantly related to intentions to join or to not join alcohol-related social gatherings?

- RQ2: Do attitudes toward behaviours, subjective norms and perceived behavioural control significantly relate to intentions to join or to not join alcohol-related social gatherings?

- RQ3: Do intentions to join or to not join alcoholrelated social gatherings significantly relate to self-reported behaviours of joining alcohol-related social gatherings?

\section{An Overview of Study 1 and Study 2}

Study 1 assessed TPB components for joining and Study 2 examined TPB components for not joining. Then, a week later, participants in both studies indicated how many alcohol-related social gatherings were accessible to them and how many of those they participated in during the preceding week. A pilot study provided a list of behavioural outcomes (five types of outcomes) and important other people (three reference groups of coworkers, supervisors and family members), that was necessary for creating the attitudinal component and the normative component measures of TPB. Some of the pilot study findings were reported in a previous study (Lee et al., 2007). Many of the outcomes were similar for joining and for not joining in that the outcomes of not joining pertained to losing opportunities to obtain the outcomes of joining. Thus, each behavioural outcome was treated as one obtainable because of joining alcohol-related social gatherings for Study 1 and as one unobtainable because of not joining alcohol-related social gatherings for Study 2. For example, participants in Study 1 rated likelihood and evaluative importance of obtaining helpful information as an outcome of joining alcohol-related social gatherings, whereas participants in Study 2 rated likelihood and evaluative importance of missing an opportunity to obtain helpful information as an outcome of not joining alcoholrelated social gatherings. In no case did participants from one study participate in another study. All questionnaires were written in Korean.

\section{Study 1}

This study tested the relative importance of the five types of behavioural outcomes for the relationship 
between attitudes toward joining alcohol-related social gatherings and intentions to join alcohol-related social gatherings (RQ1). The effects of subjective norms and perceived behavioural control on intentions (RQ2) and the relationship between intentions to join and the selfreported behaviour of joining alcohol-related social gatherings were also examined (RQ3).

\section{Study 1 Method \\ Participants}

One hundred and eight Korean employees (93.52\% men, mean age $=31.32$, ranging from 22 to 43 years old) of various civil engineering companies participated in the first part of the study, and 106 of them also participated in the follow-up part of the study. The participants and their companies were located in Seoul and its satellite cities in South Korea. They had worked in their companies for an average of 57.74 months $(S D=$ 43.58). Of the participants, 19 had high school degrees, 88 had college or graduate degrees, and one did not indicate his education level. Because the lack of women participants made it difficult to examine meaningful gender differences and generalise the findings to women, the main analyses used data from 101 men participants only. But gender and other demographic information variables did not affect any of the findings reported below. Thus, such analyses were not reported.

\section{Measures}

All of the measures used a 5-point scale (e.g., $1=$ strongly disagree, $5=$ strongly agree), unless otherwise mentioned. Intentions to join were measured with six items (e.g., 'I intend to join alcohol-related social gatherings'). Perceived behavioural control regarding joining was measured with four items (e.g., 'It is easy for me to join alcohol-related social gatherings').

Subjective norms were obtained by multiplying normative beliefs with motivation to comply and summing the products. For each of three reference groups (coworkers, supervisors and family members), three items assessed normative beliefs (e.g., 'My coworkers want me to join alcohol-related social gatherings', 'My supervisors expect me to join alcohol-related social gatherings', 'My family prefers me to join alcohol-related social gatherings') and three items assessed motivation to comply (e.g., 'I want to do what my coworkers want me to do', 'I want to do what my supervisors prefer me to do', 'I want to do what my family expects me to do'). Subjective norms for coworkers and subjective norms for supervisors were highly correlated, $r(99)=.87, p<$ .001 , and thus were combined to form subjective norms for work colleagues. Subjective norms for work colleagues and subjective norms for family were not significantly related to each other, $r(99)=.06, p=.56$.

Values for attitudes toward behaviour were obtained by first multiplying the likelihood of each behavioural outcome (anchored with $1=$ not at all likely and $5=$ extremely likely for, e.g., 'joining alcohol-related social gatherings results in improving my work group climate') and the evaluation of the outcome (anchored with $-2=$ extremely bad and 2 = extremely good for, e.g., 'improving my work group climate is___'). Then, the products of the likelihood and evaluation scores were summed for each category to form five types of attitudes toward joining alcohol-related social gatherings. Four outcomes were used for supervisor-subordinate relationship category and three outcomes were used for each of the other categories. For example, forming a sense of unity in a work group was one of the outcomes for group category; obtaining information helpful for workplace life for work-life category, being uncomfortable with supervisors for supervisor-subordinate relationship category, depth of conversation for conversation category and drinking beyond one's capacity for drinking category.

For the follow-up study one week later, the participants indicated that, on average, there had been 1.62 alcohol-related social gatherings $(S D=1.11$, Median $=$ 1 , Mode $=1)$ in the previous week and that they had attended 1.41 gatherings $(S D=1.01$, Median $=1$, Mode $=1$ ). Among the 100 participants who participated in the follow-up study, 83 participants indicated that they were given opportunities for alcohol-related social gatherings. Among those 83 participants, 67 participants did not miss any social gathering with their coworkers (i.e., a $100 \%$ participation rate), 7 had a $50 \%$ participation rate, 3 had a $66.67 \%$ participation rate, 3 had a $33.33 \%$ participation rate, 2 had a $75 \%$ participation rate and 1 had a $0 \%$ participation rate. When asked if the previous week was typical, 71 participants indicated 'yes,' 18 indicated 'no' and 11 did not answer the question. Whether the week was typical or not did not differentiate the participation rate, $t(80)=0.40, p=.69$; the number of alcohol-related social gathering opportunities, $t(86)=$ $0.27, p=.79$; or the number of alcohol-related social gatherings that they joined, $t(86)=0.27, p=.79$. Table 1 shows reliabilities, means, standard deviations, and correlations of the variables.

Rather than the raw number of times people participated in alcohol-related social gatherings, the participation rate or percentage (i.e., the number of instances of participation in alcohol-related social gatherings divided by the number of available alcohol-related social gatherings) was used to indicate the behaviour of joining alcohol-related social gatherings for a number of reasons. First, when the raw count was used, not going to alcohol-related social gatherings because no social gathering was available was not differentiated from not going to social gatherings even when social gatherings were available. Second, it was necessary to consider the number of available social gatherings as a base rate for differentiating people with the same number of instances of participation (e.g., three instances of participation indicated different participation rates if an individual had three 


\section{Table 1}

Reliabilities, Means, and Standard Deviations and Correlations of Study 1

\begin{tabular}{|c|c|c|c|c|c|c|c|c|c|c|}
\hline Study 1 & 1 & 2 & 3 & 4 & 5 & 6 & 7 & 8 & 9 & 10 \\
\hline 1. Group-related attitudes & $(.84)$ & & & & & & & & & \\
\hline 2. Work-life related attitudes & $.44^{* * *}$ & $(.75)$ & & & & & & & & \\
\hline 3. Supervisor-subordinate relationship-related attitudes & $.21 *$ & .31 & $(.86)$ & & & & & & & \\
\hline 4. Conversation-related attitudes & $.57^{* * *}$ & $.24^{*}$ & .02 & $(.68)$ & & & & & & \\
\hline 5. Drinking-related attitudes & .09 & .12 & $.53 * * *$ & .05 & $(.77)$ & & & & & \\
\hline 6. Subjective norms from work colleagues & $.41 * * *$ & $.21 *$ & -.01 & $.35 * * *$ & -.07 & $(.95)$ & & & & \\
\hline 7. Subjective norms from family & .13 & .18 & .21 & $.20^{*}$ & -.01 & .06 & $(.85)$ & & & \\
\hline 8. Perceived behavioural control & $.49^{* * *}$ & $.20^{*}$ & .19 & $.37^{* * *}$ & $.31 * *$ & $.39 * * *$ & -.03 & $(.83)$ & & \\
\hline 9. Intentions to join & $.55^{* * *}$ & $.30 * * *$ & $.22^{*}$ & $.35 * * *$ & .19 & $.43^{* * *}$ & .13 & $.64 * * *$ & $(.98)$ & \\
\hline 10. Participation rate & .11 & .09 & .14 & .18 & .00 & -.04 & .17 & .05 & .14 & - \\
\hline$d f$ & 99 & 99 & 99 & 99 & 99 & 99 & 99 & 99 & 99 & 81 \\
\hline$M$ & 4.02 & 0.48 & -2.86 & 3.72 & -3.49 & 12.69 & 2.96 & 3.65 & 3.63 & 90.36 \\
\hline$S D$ & 2.35 & 1.82 & 2.13 & 1.96 & 2.95 & 3.55 & 3.28 & 0.65 & 0.85 & 21.44 \\
\hline
\end{tabular}

Notes. ${ }^{* *} p<.001,{ }^{* *} p<.01,{ }^{*} p<.05$

Reliabilities (Cronbach's $\alpha$ ) are reported in parentheses on the diagonal.

Participation rate: Frequencies of joining alcohol-related social gatherings at time 2 for those who indicated alcohol-related social gatherings were available to them.

invitations to alcohol-related social gatherings than if another individual had six invitations to alcohol-related social gatherings in one week). Third, when the raw number of instances of participation was used, there was a greater likelihood of an extreme score (i.e., outlier) that could alter the results.

\section{Study 1 Results}

Three research questions were asked regarding which types of attitudes toward joining alcohol-related social gatherings would be important for intentions (RQ1), whether the three TPB components would predict intentions (RQ2) and whether intentions would predict behaviour (RQ3). To address RQ1 and RQ2, a multiple regression analysis was conducted with the eight predictors for intentions to join. For this regression analysis, no second (or higher) order interactions or curvilinear relationships were observed. For example, the interactions among the TPB components did not explain significant amount of variance in intentions, $F_{\text {change }}(12$, $80)=0.93, p=.52, R_{\text {change }}^{2}=.06$. Thus, any higher order relationships among the TPB components are not reported below. Finally, to address RQ3, the correlation between intentions to join and self-reported behaviour was examined. Table 2 reports the regression results.

\section{TPB Components and Intentions}

The multiple regression analysis yielded a significant result, $F(8,92)=12.42, p<.001$, adj. $R^{2}=.48$, as shown in Table 2. Among the five types of attitudes, grouprelated attitudes toward joining was a significantly positive predictor for intentions to join alcohol-related social gatherings; with a higher likelihood and evaluative importance of obtaining group-related outcomes, the participants had stronger intentions to join. Subjective norms from work colleagues and subjective norms from family were not statistically significant for intentions to join. By contrast, perceived behavioural control regarding joining was significant for intentions to join alcohol-related social gatherings.

\section{Intention and Behaviour}

Intentions to join were not significantly related to participation rate one week later, $r(81)=.14, p=.21$ (see Table 1). The lack of significant relationship between intentions and self-reported behaviour needs to be interpreted cautiously, as range restrictions existed (i.e., $81 \%$ of the participants had a $100 \%$ participation rate). A closer inspection of the data indicated that $73.27 \%(n=74)$ of the participants scored higher than the midpoint (3) of the scale measuring intentions to join. Of those participants, 62 participants in the follow-up study indicated at least one gathering was available to them in the previous week. Of those 62 participants, $83.87 \%(n=52)$ had a $100 \%$ participation rate and only one person had a $0 \%$ participation rate. In contrast, $16.83 \%(n=17)$ of the participants scored lower than the midpoint (3) of the scale measuring intentions to join. Of these participants, 13 participants in the follow-up study indicated at least one gathering was available to them in the previous week. Of those 13 participants, $76.92 \%(n=10)$ had a $100 \%$ participation rate and none had a $0 \%$ participation rate.

\section{Study 2}

The main purpose of Study 2 was to examine how TPB components concerning the behaviour of not joining alcohol-related social gatherings would be related to 
Table 2

Multiple Regression Analysis Results of Study 1 (Intentions to Join Alcohol-Related Social Gatherings)

\begin{tabular}{|c|c|c|c|}
\hline & B & SE & $\beta$ \\
\hline Group-related attitudes & 0.09 & 0.04 & $.24^{*}$ \\
\hline Work-life related attitudes & 0.02 & 0.04 & .05 \\
\hline Supervisor-subordinate relationship-related attitudes & 0.02 & 0.04 & .05 \\
\hline Conversation-related attitudes & -0.02 & 0.04 & -.05 \\
\hline Drinking-related attitudes & 0.00 & 0.03 & .00 \\
\hline Subjective norms from work colleagues & 0.04 & 0.02 & .15 \\
\hline Subjective norms from family & 0.03 & 0.02 & .10 \\
\hline \multirow[t]{2}{*}{ Perceived behavioural control } & 0.61 & 0.12 & $.47^{* * *}$ \\
\hline & \multicolumn{3}{|c|}{$F(8,92)=12.42, p<.001$, adj. $R^{2}=.48$} \\
\hline
\end{tabular}

Note. ${ }^{* * *} p<.001,{ }^{* *} p<.01,{ }^{*} p<.05$

intentions to not join. If joining and not joining alcoholrelated social gatherings constitute two opposites of one behaviour, the findings of Study 1 would be replicated in Study 2. Those outcomes associated with joining, however, may not be significantly associated with intentions to not join. For example, when individuals intend to not join alcohol-related social gatherings, it may be because they favour avoiding excessive drinking rather than losing opportunities to improve a work group climate. Thus, the three research questions examined in Study 1 will be examined again in Study 2, but the focus will be on the TPB components measured on the behaviour of not joining.

\section{Study 2 Method}

\section{Participants}

One hundred and seven Korean employees (91.59\% men, mean age $=33.47$, ranging from 22 to 62 years old) of various civil engineering companies participated in the first part of the study, and 104 of them also participated in the follow-up of the study. The participants and their companies were located in Seoul and its satellite cities in South Korea. The workers were employed at their companies for 59.71 months on average $(S D=$ 79.89). Of the participants, 16 had high school degrees, 78 had college or graduate degrees and 13 did not indicate their education levels. Because the lack of women participants made it difficult to examine meaningful gender differences and generalise the finding to women, the main analyses used data from 98 men participants only. But gender and other demographic information variables did not affect any of the findings reported below. Thus, such analyses were not reported.

\section{Measures}

All of the measures used a 5-point scale (e.g., $1=$ strongly disagree, 5 = strongly agree), unless otherwise mentioned. Intentions, perceived behavioural control, subjective norms and attitudes toward behaviours were measured in a similar way as in Study 1. The major difference from Study 1 was that the measurement items in
Study 2 pertained to not joining alcohol-related social gatherings. Intentions to not join were measured with six items (e.g., 'I do not intend to join alcohol-related social gatherings'). Perceived behavioural control regarding not joining was measured with four items, including 'It is easy for me to not join alcohol-related social gatherings'. Subjective norms regarding not joining were measured with items such as 'My coworkers want me to not join alcohol-related social gatherings' for normative beliefs and 'I want to do what my coworkers want me to do' for motivation to comply. Subjective norms were obtained by multiplying normative beliefs with motivation to comply and summing the products. Subjective norms for coworkers and subjective norms for supervisors were highly correlated, $r(96)=.92, p<$ .001 , and were combined to form subjective norms for work colleagues. Subjective norms for work colleagues and subjective norms from family were not significantly related to each other, $r(96)=.16, p=.12$.

Attitudes toward not joining were measured with items indicating the outcomes of not joining alcoholrelated social gatherings. The findings of a pilot study showed that the many outcomes of not joining alcoholrelated social gathering were categorised as avoiding or missing outcomes of joining alcohol-related social gathering. The outcomes of joining alcohol-related social gatherings used in Study 1 were also used in Study 2. For example, the outcome of 'improving my work group climate' used in Study 1 was transformed to 'missing an opportunity to improve my work group climate' for Study 2. Missing an opportunity to improve a work group climate was one of the outcomes for group category (three items); creating an impression as an individualistic person for work-life category (three items), not having an uncomfortable time with supervisors for supervisor-subordinate relationship category (four items), missing an opportunity to have in-depth conversations with coworkers for conversation category (three items) and avoiding drinking against one's will for drinking category (three items). Attitudes toward behaviour (i.e., attitudes toward 
not joining) were obtained by first multiplying the likelihood of each behavioural outcome (anchored with $1=$ not at all and $5=$ extremely likely) and the evaluation of the outcome (anchored with $-2=$ extremely bad and $2=$ extremely good) across 16 outcomes (e.g., for likelihood of behavioural outcomes, 'joining alcohol-related social gatherings results in missing opportunity to improve my work group climate'; for evaluation of the outcomes, 'missing opportunity to improve my work group climate is __'). Then, the products of the likelihood and evaluation scores were summed for each of the five categories to form five types of attitudes toward not joining alcoholrelated social gatherings.

For the follow-up study, participants indicated that there were 2.38 alcohol-related social gatherings on average $(S D=1.09$, Median $=2$, Mode $=2)$ in the previous week and that they went to 2.25 gatherings $(S D=$ 1.01, Median $=2$, Mode $=2$ ). Among the 98 participants in the follow-up study, 95 participants indicated that they were given opportunities for alcohol-related social gatherings. Among those 95 participants, 85 participants did not miss any gathering with their coworkers (i.e., a $100 \%$ participation rate), 4 had a $75 \%$ participation rate, 3 had a $50 \%$ participation rate, 2 had a $66.67 \%$ participation rate and 1 had a $60 \%$ participation rate. When asked if the previous week was typical, 71 participants indicated 'yes', 19 indicated 'no', and 5 did not answer the question. Whether the week was typical or not did not differentiate the participation rate, $t(87)=0.30, p=.77$, or the number of alcohol-related social gathering opportunities, $t(88)=1.81, p=.07$. Whether the week was typical or not, however, differentiated the raw number of alcohol-related social gatherings that the participants joined, $t(88)=2.09, p=.04$. Participants who indicated the previous week as typical reported a higher number of alcohol-related social gatherings that they joined $(M$ $=2.38, S D=1.02)$ than did those who indicated the previous week as not typical $(M=1.84, S D=0.90)$. Table 3 shows reliabilities, means, standard deviations, and correlations of the variables.

\section{Study 2 Results}

The three research questions were examined using multiple regression and correlation analyses. When conducting the multiple regression analysis, a secondorder interaction was observed, but no other higher order interactions or curvilinear relationships were observed. The predictor variables were mean-centred before multiplying them to create second-order interaction terms. Hierarchical multiple regression analysis was conducted with the TPB components in the first block and the second-order product terms of the predictor variables in the second block. The second-order predictors were created by focusing on the interactions of each type of attitudes by each type of subjective norms and perceived behavioural control. But the interactions of attitudes by subjective norms from family and also the interactions of attitudes by perceived behavioural control were not significant and did not affect the overall pattern of multiple regression analysis results. Thus, only the interactions of attitudes by subjective norms for work colleagues were included in the final analysis. The results are shown in Table 4 .

\section{TPB Components Regarding Not Joining and Intention to Not Join}

First-order predictors. For the relationship between different types of attitudes toward not joining and intentions

Table 3

Reliabilities, Means, and Standard Deviations and Correlations of Study 2

\begin{tabular}{|c|c|c|c|c|c|c|c|c|c|c|}
\hline Study 2 & 1 & 2 & 3 & 4 & 5 & 6 & 7 & 8 & 9 & 10 \\
\hline 1. Group-related attitudes & $(.89)$ & & & & & & & & & \\
\hline 2. Work-life related attitudes & .16 & $(.67)$ & & & & & & & & \\
\hline 3. Supervisor-subordinate relationship-related attitudes & .12 & -.08 & $(.90)$ & & & & & & & \\
\hline 4. Conversation-related attitudes & $.71^{* * *}$ & -06 & -.01 & $(.78)$ & & & & & & \\
\hline 5. Drinking-related attitudes & -.16 & .02 & $.33^{* *}$ & -.23 & $(.74)$ & & & & & \\
\hline 6. Subjective norms from work colleagues & -.15 & .08 & -.01 & -.21 & .09 & $(.91)$ & & & & \\
\hline 7. Subjective norms from family & -.18 & -.02 & $.29 * *$ & $-.33^{* *}$ & $32 * *$ & .16 & $(.92)$ & & & \\
\hline 8. Perceived behavioral control & -.10 & .11 & -.07 & -.17 & -.15 & .09 & -.17 & $(.70)$ & & \\
\hline 9. Intentions to Not join & .09 & .00 & .08 & .09 & .09 & .12 & -.09 & .04 & $(.89)$ & \\
\hline 10. Participation rate & -.06 & .04 & .02 & -.05 & .18 & .03 & .02 & -.02 & .02 & - \\
\hline$d f$ & 96 & 96 & 96 & 96 & 96 & 96 & 96 & 96 & 96 & 92 \\
\hline M & -2.87 & -0.30 & 0.47 & -2.29 & 2.84 & 7.54 & 12.44 & 3.14 & 2.94 & 96.21 \\
\hline SD & 2.46 & 1.99 & 3.02 & 2.76 & 2.56 & 2.70 & 6.11 & 0.62 & 0.69 & 11.60 \\
\hline
\end{tabular}


(RQ1), the results showed that none of the attitude types were statistically significant for intentions to not join. Answering RQ2, subjective norms and perceived behavioural control did not explain significant variation in intentions, as shown in Table 4 . None of the predictors were significant for intentions to not join.

Second-order predictors. The interaction between group-related attitudes and subjective norms from work colleagues was the only significant one among the second-order predictors. Simple regression analyses were conducted at various points of subjective norms from work colleagues. When subjective norms from work colleagues were at $1 S D$ below its mean, the group-related attitudes about not joining were not significantly related to intentions to not join, $B=0.01, S E=0.05, t=0.22, p$ $=.83$. The relationship between the group-related attitudes about not joining and intentions to not join became stronger when subjective norms from work colleagues were at its mean, $B=0.09, S E=0.05, t=1.85, p$ $=.07$. When subjective norms from work colleagues were at $1 S D$ above its mean, the group-related attitudes about not joining were significantly related to intentions to not join, $B=0.15, S E=0.05, t=3.09, p=.003$.

\section{Intention and Behaviour}

Intentions to not join alcohol-related social gatherings would be expected to relate negatively to participation rate a week later. Intentions to not join were not significantly related to participation rates, $r(92)=.02, p=.82$ (see Table 3). The lack of significant relationships between intentions and self-reported behaviour may be attributed to the lack of variance in behaviour. A closer inspection of the data indicated that $40.82 \%(n=40)$ of the participants scored higher than the midpoint (3) of the scale measuring intentions to not join. Of those participants, 39 participants also participated in the follow-up study and indicated that at least one gathering was available to them in the previous week. Of the 39 participants, $89.74 \%(n=35)$ had a $100 \%$ participation rate and none had a $0 \%$ participation rate. By contrast, $45.92 \%(n=45)$ of the participants scored lower than the midpoint (3) of the scale measuring intentions to not join. Of these 45 participants, 44 participated in the follow-study and indicated that at least one gathering was available to them in the previous week. Of the 44 participants, $90.91 \%(n=40)$ had a $100 \%$ participation rate and none had a $0 \%$ participation rate.

\section{Discussion}

The findings from the current research may indicate that joining or not joining alcohol-related social gatherings are not mere behavioural opposites, predictable by the presence or absence of the same behavioural outcomes. These two aspects of the behaviour seem to require assessment of different behavioural outcomes or different assessments of the same behavioural outcomes. Group-related attitudes and perceived behavioural controls regarding joining and not joining were differen-

Table 4

Moderated Multiple Regression Analysis Results of Study 2 (Intentions to Not Join Alcohol-Related Social Gatherings)

\begin{tabular}{|c|c|c|c|}
\hline & B & SE & $\beta$ \\
\hline \multicolumn{4}{|l|}{ First block } \\
\hline Group-related attitudes & 0.02 & 0.04 & .06 \\
\hline Work-life related attitudes & -0.01 & 0.04 & -.02 \\
\hline Supervisor-subordinate relationship-related attitudes & 0.02 & 0.03 & .07 \\
\hline Conversation-related attitudes & 0.02 & 0.04 & .08 \\
\hline Drinking-related attitudes & 0.03 & 0.03 & .13 \\
\hline Subjective norms from work colleagues & 0.03 & 0.03 & .16 \\
\hline Subjective norms from family & -0.01 & 0.01 & -.13 \\
\hline \multirow[t]{2}{*}{ Perceived behavioural control } & 0.05 & 0.12 & .05 \\
\hline & \multicolumn{3}{|c|}{$F(8,89)=0.72, p=.67$, adj. $R^{2}=.00$} \\
\hline \multicolumn{4}{|l|}{ Second block } \\
\hline Group-related attitudes $\times$ Subjective norms from work colleagues & 0.04 & 0.01 & $.32 * *$ \\
\hline Work-life related attitudes $\times$ Subjective norms from work colleagues & 0.02 & 0.01 & .16 \\
\hline Supervisor-subordinate relationship-related attitudes $\times$ Subjective norms from work colleagues & -0.01 & 0.01 & -.15 \\
\hline \multirow[t]{2}{*}{ Drinking-related attitudes $\times$ Subjective norms from work colleagues } & 0.01 & 0.01 & .13 \\
\hline & \multicolumn{3}{|c|}{$F_{\text {change }}(4,85)=4.01, p=.005, R_{\text {change }}=.15$} \\
\hline
\end{tabular}

Note ${ }^{* *} p<.01,{ }^{*} p<.05$

As shown in Table 3, group-related attitudes and conversation-related attitudes were highly correlated with one another, which may raise concerns for multicollinearity. The highest variation inflation factor (VIF) was 2.46 among the first-order predictors. This VIF level was lower than the traditional rule of thumb threshold value (e.g., 10) and more stringent threshold value (e.g., 6) (Cohen, Cohen, West, \& Aiken, 2003). For second-order predictors, however, the interaction terms involving conversation-related attitudes were not included in the second block to avoid multicollinearity possibilities. Whether or not the interaction terms involving conversation-related attitudes were included in the second block did not affect any of the findings reported above. For example, conversation-related attitudes were not a significant predictor of intentions to not join and any interaction terms involving conversation-related attitudes were not significant. 
tially related to intentions to join and to not join. Engaging and not engaging in a behaviour may not be mirror images of each other.

\section{Implications for TPB Components}

Attitudes toward behaviour. TPB posits that likelihood and evaluative importance of achieving certain behavioural outcomes (i.e., attitudes toward engaging in a behaviour) are important for forming intentions to engage in a behaviour. The current study additionally examined how likelihood and evaluative importance of not achieving such outcomes (i.e., attitudes toward not engaging in a behaviour) are related to intentions to not engage in the behaviour. For the cases of group-related attitudes toward joining (e.g., improving a work group climate) and intentions to join, the overall findings indicated that the more likely and more important improving a work group climate was, for example, as an outcome of joining, the stronger the intentions people would have toward joining. This finding is in line with TPB. Conversely, for the case of the group-related attitudes toward not joining (e.g., losing an opportunity to improve a work group climate) and intentions to not join, there was no significant relationship between attitudes and intentions.

The relationship between the group-related attitudes toward not joining and intentions to not join depended on subjective norms from work colleagues in such a way that the more likely and more important missing out on an opportunity to improve a work group climate was as an outcome of not joining, the stronger individuals' intentions were about not joining alcohol-related social gatherings among individuals with strong subjective norms from work colleagues. This finding about grouprelated attitudes toward not joining may seem counterintuitive. A possible explanation is that unlike TPB, which presupposes that evaluations of the likelihood and importance of behavioural outcomes precede formation of behavioural intentions, individuals in the current study might have formed intentions to not join beforehand and then evaluated the behavioural outcomes of not joining. That is, individuals, who have stronger perceptions that their work colleagues expected them to not join, might have considered losing opportunities to improve a work group climate as the result of their intentions to not join rather than as the reason for intentions to not join. Especially for applying TPB to understanding individuals' not engaging in a behaviour, further research needs to examine how evaluations of behavioural outcomes relate to intentions to not engage.

Subjective norms. Unlike many other studies with TPB, the current study did not find subjective norms to be significant predictors of intentions to join and intentions to not join. These findings are at odds with other study on the similar topic, Korean undergraduates' intentions to join and to not join alcohol-related social gatherings (Park \& Lee, 2009). Meta-analysis showed that although subjective norms had a weaker relationship with intentions than did attitudes, subjective norms were predictive of intentions across various studies (Armitage \& Conner, 2001). There have been exceptions, however; subjective norms were not statistically significant for amount of self-reported drinking (Murgraff et al., 2001) and for engaging in heavy drinking (Norman et al., 1998). Due to the collectivistic characteristics of Korean culture, norms can play an important part for Koreans' behaviours. Studies showed that normative influence was important for Koreans' behavioural intentions and behaviours (e.g., Jo, Lee, Ahn, \& Jung, 2003; Lee \& Green, 1991; Myeong \& Crawley, 1993; Park, 1998). Other studies, however, found that normative factors were not significant for Koreans' behavioural intentions (e.g., Park \& Levine, 1999; Yoo, 1996). The findings of the current study, along with past studies, may indicate that subjective norms are not universally important for intentions and behaviours.

The current study found that subjective norms from work colleagues were significant only as a moderator for the relationship between group-related attitudes toward not joining and intentions to not join alcohol-related gatherings. Park and Smith (2007) showed that subjective norms were a moderator for the relationship between attitudes and intentions to talk with family about organ donation. Individuals may be more likely to behave in accordance with their attitudes when their attitudes are consistent with in-group norms than when their attitudes are inconsistent with in-group norms (White, Hogg, \& Terry, 2002). For not engaging in a behaviour such as not joining alcohol-related social gatherings with work colleagues, the current finding indicated that attitudes toward not joining became relevant to intentions to not join only when work colleagues do not strongly endorse alcoholrelated social gatherings.

Perceived behavioural control. Perceived behavioural control regarding joining was significantly related to intentions to join, while perceived behavioural control regarding not joining was not significantly related to intentions to not join. This finding raises the question of whether joining and not joining alcohol-related social gatherings are both volitional behaviours for Korean working adults. Compared to Korean undergraduates who have not experienced corporate culture yet, Korean working adults have less positive attitudes about their past decision not to join a drinking party and indicate lower self-efficacy in joining drinking parties (Lee, Park, Lee, Kim, \& Kim, 2006). It may be possible that not joining alcohol-related social gatherings may not be as volitional a behaviour for Korean working adults as for other populations, such as undergraduates and/or people in individualistic cultures. If so, it is also possible that working adults' perceived control of joining alcohol-related social gatherings may not be a 'real' 
control, but a misconceived one (i.e., illusion). Considering that perceived behavioural control was significantly correlated with subjective norms, Korean working adults may internalise group pressure to comply, not clearly distinguishing between their own internal decisions and external ones contributed by their reference groups.

Korean culture is characterised as higher in power distance than western cultures (Hofstede, 1980). In high power distance cultures, greater inequalities exist between people in lower status and those in higher status, and top-down decision-making is more prevalent and accepted. In Korean society, which prioritises hierarchy and custom, it is important to maintain smooth social and work relationships with coworkers and immediate supervisors (Cho \& Yoon, 2002). If supervisors pressure subordinates to join social gatherings it may not be easy for the subordinates to resist such pressure and voice their opinions. However, if individuals do not perceive much control in joining social gatherings and drinking alcohol is not a volitional behaviour, it can have consequences on health. An open communication climate where workers can express their opinions and exchange ideas freely is important for successful implementation of innovative approaches (Lee, Park, \& Choi, in press). If Korean companies and working adults want to improve their health by reducing excessive alcohol consumption, health campaigns in the workplace may need to focus on fostering an open communication climate and help workers to increase their control regarding joining and not joining 회식.

Intention and behaviour. Intentions were not significantly related to self-reported behaviour in Study 1 as well as in Study 2 of the current research. Various meta-analyses provided strong evidence that intentions are predictive of behaviours (Sheeran, 2002; Webb \& Sheeran, 2006). Sheeran (2002) and Webb and Sheeran (2006) discussed potential moderators for the intention-behaviour relation such as behaviour characteristics and measurement artefacts. Because the findings of the current research are not consistent with the results of the meta-analyses, it is necessary to consider some features of the current study that might have contributed to weakening the correlation between intentions and behaviour. First, participation in alcohol-related social gatherings with work colleagues could be a repeatedly performed behaviour in a stable context that was less likely to be under the control of intentions. It is possible that, for Korean adults, participation in alcohol-related social gatherings with work colleagues could be almost a habitual or frequent behaviour, as indicated in Lee et al.'s study (2007), which reported that many of their Korean participants drank with work colleagues twice a month $(26.4 \%)$, four times a month $(24.1 \%)$, or once a month $(18.4 \%)$. Especially in the case of Study 1 of the current research, the nonsignificant correlation between intentions and behaviour could be due to a restriction in range of participation rate (i.e., an extremely high rate of participation in alcohol-related social gatherings with work colleagues) because this behaviour is one that is consistently and habitually performed, regardless of one's intention.

Second, an interesting aspect of Study 2 of the current research is the disinclined actors responsible for the lack of intention-behaviour relation. Disinclined actors are those who do not intend to behave but engage in the behaviour and inclined abstainers are those who intend to behave but fail to engage in the behaviour (Sheeran, 2002). Disinclined actors and inclined abstainers are two categories of people who show inconsistency between intentions and behaviours. Sheeran (2002) noted that the inconsistency between intentions and behaviours in the past research was mainly due to inclined abstainers. It was disinclined actors, however, who were responsible for the inconsistency between intentions and behaviour in Study 2 of the current research. In Study 2, which examined intentions to not join alcohol-related social gatherings, among the participants who scored higher than the midpoint of the scale (i.e., positively inclined to not join; in other words, negatively inclined to join), $89.74 \%$ reported a $100 \%$ participation rate. Future research examining factors affecting not engaging in a behaviour may benefit from a systematic investigation of the conditions that induce more disinclined actors than inclined abstainers.

\section{Limitations and Future Directions}

The current study has a few limitations. First, the majority of the participants were men employed in the Korean civil engineering industry. Second, the current findings were based on people's self-reported behaviours and perceptions about their behaviours, rather than more objective observations and actual behaviours. Third, because the short time period (i.e., one week) between the measurement of intentions and the measurement of participation in alcohol-related social gatherings, it is possible that some of the participants simply did not have the opportunity to engage or not engage in the behaviour in that short time period. Multiple follow-up studies and/or a longer period between the first and the second parts of the study might have reduced the restriction in range and could have increased the chance for observing intention-behaviour consistency. These factors may limit generalisation of the current findings and need to be considered for future research. Considering that Korean supervisors and subordinates do not have mutually accurate perceptions of their relationship with one another (Lee, Lee, Lee, \& Park, 2005), it will be interesting for future studies to examine the perspectives of both supervisors and subordinates regarding the role of alcohol in social gatherings after work hours.

\section{Conclusion}

Many behaviours, especially in health research areas, may require understanding of both aspects of a behaviour - 
engaging and not engaging in the behaviour - in order to develop better theories and design more effective health campaigns. Additionally, rather than simply stating that attitudes are important for intentions and behaviours, it is useful to identify the relevant behavioural outcomes to which people pay attention when intending to engage and to not engage in a behaviour and to examine the relationships between specific outcome-related attitudes toward behaviours and intentions.

\section{References}

Ajzen, I. (1988). Attitudes, personality and behavior. MiltonKeynes, England: Open University Press.

Ajzen, I., \& Fishbein, M. (1980). Understanding attitudes and predicting social behavior. Englewood-Cliffs, NJ: Prentice-Hall.

Albarracín, D., Johnson, B.T., Fishbein, M., \& Muellerleile, P.A. (2001). Theories of reasoned action and planned behavior as models of condom use: A meta-analysis. Psychological Bulletin, 127, 142-161.

Armitage, C.J., \& Conner, M. (2001). Efficacy of the theory of planned behaviour: A meta-analytic review. British Journal of Social Psychology, 40, 471-499.

Chau, P.Y.K. \& Hu, P.J.-H. (2001). Information technology acceptance by individual professionals: A model comparison approach. Decision Sciences, 32, 699-719.

Cho, Y.-H., \& Yoon, J. (2002). The origin and function of dynamic collectivism: An analysis of Korean corporate culture. In C. Rowley, T.-W. Sohn, \& J. Bae (Eds.), Managing Korean business: Organization, culture, human resource and change (pp. 70-88). London: Frank Cass.

Choi, S.-H., Kim, M., \& Kim, K.-K. (2001). 서울 지역 사무직 근로자의 음주에 관한 행태 및 관련요인에 관한 조사 연[Drinking behavior and related factors among white collar workers in Seoul]. 보건교육 - 건강증진학회지 [Journal of Korean Society for Health Education and Promotion], 18, 27-44.

Codd, R.T., \& Cohen, B.N. (2003). Predicting college student intention to seek help for alcohol abuse. Journal of Social and Clinical Psychology, 22, 168-191.

Corrao, G., Bagnardi, V., Zambon, A., \& Arico, S. (1999). Exploring the dose-response relationship between alcohol consumption and the risk of several alcohol-related conditions: A meta-analysis. Addiction, 94, 1551-1573.

Fekadu, Z., \& Kraft, P. (2002). Expanding the theory of planned behaviour: The role of social norms and group identification. Journal of Health Psychology, 7, 33-43.

Godin, G., \& Kok, G. (1996). The theory of planned behavior: A review of its applications to health-related behaviors. American Journal of Health Promotion, 11, 87-98.

Hagger, M.S., Anderson, M., Kyriakaki, M., \& Darkings, S. (2007). Aspects of identity and their influence on intentional behavior: Comparing effects for three health behaviors. Personality and Individual Differences, 42, 355-367.

Hofstede, G. (1980). Culture's consequence. Beverly Hills, CA: Sage.

Hu, S.-C., \& Lanese, R.R. (1998). The applicability of the theory of planned behavior to the intention to quit smoking across workplaces in southern Taiwan. Addictive Behaviors, 23, 225-237.

Jo, H., Lee, S., Ahn, M.O., \& Jung, S.H. (2003). Structural relationship of factors affecting health promotion behaviors. Health Promotion International, 18, 229-236.

Kim, J.K. (1986). 현대 한국인의 음주양태 [Recent Drinking patterns of Koreans]. 정신건강연구 [Mental Health Research], 4, 9-19.

Kim, M.-S. (2002). Non-Western perspectives on human communication: Implications for theory and practice. Thousand Oaks, CA: Sage.

Korea National Statistical Office. (2003). 2002 년 사망원 인통 계결 과 [The results of 2002 death cause statistics survey]. Daejeon, Korea: Author.

Lee, K.S. (2009). The drinking patterns and tendencies of public officials (Master's thesis, Kyungpook National University, Daegu, Korea). Retrieved from http://www.riss.kr

Lee, C., \& Green, R.T. (1991). Cross-cultural examination of the Fishbein behavioral intention model. Journal of International Business Studies, 22, 289-305.

Lee, S.A, Park, H.S., \& Choi, J. (2011). The relationship between communication climate and elementary teachers' beliefs about alternative assessment. Journal of Pacific Rim Psychology, 5(1), 11-18.

Lee, T.S., Lee, D.W., Lee, H.E., \& Park, H.S. (2005). Superior-subordinate relationship in Korean civil engineering companies. Journal of Management in Engineering, 21, 159-163.

Lee, D.W., Park, H.S., Lee, T.S., Kim, M.K., \& Kim, Y.H. (2006). Korean working adults' and undergraduate students' attitudes towards, and self-efficacy in, joining drinking parties. Social Behavior and Personality, 34, 487-498.

Lee, D.W., Park, H.S., Lee, T.S., Kim, M.K., \& Kim, Y.H. (2007). Functions of social gatherings involving alcohol consumption with coworkers in Korea. Asian Journal of Communication, 17, 266-284.

Ministry of Health and Welfare. (2003). 2001 국민건강.영양 조사 [The results of health and nutrition survey in the year of 2001]. Gwa Chon, Korea: Author.

Murgraff, V., McDermott, M.R., \& Walsh, J. (2001). Exploring attitude and belief correlates of adhering to the new guidelines for low-risk single-occasion drinking: An application of the theory of planned behaviour. Alcohol \& Alcoholism, $36,135-140$.

Myeong, J-O., \& Crawley, F.E. (1993). Predicting and understanding Korean high school students' science-track choice: Testing the theory of reasoned action by structural equation modeling. Journal of Research in Science Teaching, 30, 381-400.

National Institute on Alcohol Abuse and Alcoholism. (1998). Alcohol and the liver: Research update. Retrieved March, 31, 2004, from http://www.niaaa.nih.gov/publications/aa42.htm Alcohol Alert No. 42. Bethesda, MD: Author

Norman, P., Bennett, P., \& Lewis, H. (1998). Understanding binge drinking among young people: An application of the Theory of Planned Behavior. Health Education Research, $13,163-169$.

Park, H.S. (1998). The theory of reasoned action and self-construals in predicting intention of studying among Korean 
college students. Communication Research Reports, 15, 267-279.

Park, H.S., \& Lee, D.W. (2009). A test of theory of planned behavior in Korea: Participation in alcohol-related social gatherings. International Journal of Psychology, 44, 418-433.

Park, H.S., \& Levine, T.R. (1999). The theory of reasoned action and self-construals: Evidence from three cultures. Communication Monographs, 66, 199-218.

Park, H.S., \& Smith, S.W. (2007). Distinctiveness and influence of subjective norms, personal descriptive and injunctive norms, and societal descriptive and injunctive norms on behavioral intent: A case of two behaviors critical to organ donation. Human Communication Research, 33, 194-218.

Sheeran, P. (2002). Intention-behavior relations: A conceptual and empirical review. In W. Stroebe, \& M. Hewstone, (Eds.), European review of social psychology (vol. 12, pp. 1-34). Chichester, England: John Wiley \& Sons.

Sheppard, B.H., Hartwick, J., \& Warshaw, P.R. (1988). The theory of reasoned action: A meta-analysis of past research with recommendations for modifications and future research. Journal of Consumer Research, 15, 325-343.
Sung, K., \& Kim, M. (2009). A comparison of alcohol-related problems between men and women in Korean university students. Korean Journal of Health Education and Promotion, 26, 85-95.

Webb, T.L., \& Sheeran, P. (2006). Does changing behavioral intentions engender behavior change?: A meta-analysis of the experimental evidence. Psychological Bulletin, 132, 249-268.

White, K.M., Hogg, M.A., \& Terry, D.J. (2002). Improving attitude-behavior correspondence through exposure to normative support from a salient ingroup. Basic and Applied Social Psychology, 24, 91-103.

Yoo, H. (1996). Attitudes, subjective norms, and beliefs of Korean nursing students as predictors of intentions to care for HIV disease patients: A test of theory of reasoned action. Dissertation Abstracts International, 57(10), 6183B. (UMI No. 9709111)

Yun, D., \& Park, H.S. (2010). Culture and the Theory of Planned Behavior: Organ donation intentions in Americans and Koreans. Journal of Pacific Rim Psychology, 4(2), 130-137. 